\title{
Quantitative evaluation of reconstruction methods after gastrectomy using a new type of examination: digestion and absorption test with stable isotope ${ }^{13} \mathrm{C}$-labeled lipid compound
}

\author{
Makoto Takase, Yoshinobu Sumiyama, and Jiro Nagao \\ The Third Department of Surgery, Toho University School of Medicine, Ohashi Hospital, 2-17-6 Ohashi, Meguro-ku, Tokyo 153-8515, Japan
}

\begin{abstract}
Background. Digestive and absorptive disorders may negatively influence patients' nutrition, thus resulting in weight loss after gastrectomy. A relationship thus seems to exist between the fat absorptive function and body weight after gastrectomy; however, so far there has been no evidence to prove this hypothesis. Therefore, in this study we evaluated fat absorption ability using a stable isotope, ${ }^{13} \mathrm{C}$-trioctanoin, based on the range of the gastrectomy and the method of reconstruction, and we also determined the feasibility of this test.

Methods. Among patients who had undergone gastrectomy for gastric cancer, 40 patients who had been operated on between 1 and 3 years previously were evaluated. Ten patients had undergone the double-tract (DT) method, and 10 patients had received the Roux-en-Y (RY) method after a total gastrectomy. Twenty patients who had undergone the Billroth I (BI) method after a distal gastrectomy were the control group. In addition, 10 volunteers formed a healthy control group for the ${ }^{13}$ C-trioctanoin test. We also examined other six factors related to nutrition after gastrectomy.

Results. The ${ }^{13} \mathrm{C}$-trioctanoin test showed, in relation to the reconstruction procedure, the highest average peak of fat absorption in the BI group (which had food passage through the duodenum), followed by the average peak of fat absorption in the DT group and the RY groups. In a comparison of duration, at $60 \mathrm{~min}$ and $90 \mathrm{~min}$ after administration, the BI group and DT group showed a significantly higher level than the RY group. The peaking time (average time at peak level) showed a significant difference between the RY group and the other groups. The absorption amount at an early stage of absorption and the percent $(\%)$ dose showed a significant difference between the RY group and the other groups. The RY group had significantly lower fat absorption than the healthy controls. Conclusion. According to this study, which evaluated fat absorption after different reconstructive procedures after gastrectomy, the procedure that accommodated for the passage of food through the duodenum showed better results for the
\end{abstract}

Offprint requests to: $\mathrm{M}$. Takase

Received: September 30, 2002 / Accepted: April 1, 2003 absorption of medium-chain triglycerides, and the patients also showed a better physiological state.

Key words ${ }^{13} \mathrm{C}$-Trioctanoin $\cdot$ Fat absorption test $\cdot$ Nonradioactive breath test $\cdot$ Reconstructive operation - Gastric cancer

\section{Introduction}

As gastrectomy has become increasingly safer to perform in recent years, it is important to improve the patients' postoperative quality of life (QOL). Negative factors in the QOL include reflux esophagitis, dumping syndrome, and digestive and absorptive disorders. In particular, digestive and absorptive disorders may have a negative influence on the patients' nutrition status, resulting in weight loss. To select surgical reconstructions that provide better physiological function, various tests have been performed. Conventional methods such as the pancreatic function diagnostic test (PFD) and Dxylose absorption test are not direct examinations. Although examinations utilizing ${ }^{14} \mathrm{C}$ compounds are direct examinations, they have, so far, only been performed in a limited number of patients. In addition, such examinations use radioactive isotopes, and therefore have recently fallen out of favour. Up to the present, many reports have concluded that reconstruction methods which allow for the passage of food through the duodenum are more favorable, but this hypothesis has not yet been proven. In this study, the ${ }^{13} \mathrm{C}$-trioctanoin absorption test, which uses a safe stable isotope, and a new direct fat absorption test, were performed in order to prove this hypothesis. The findings of digestion and absorption tests using the stable isotope ${ }^{13} \mathrm{C}$-trioctanoin, based on reconstructive methods after gastrectomies for gastric cancer, have not yet been reported. The ${ }^{13} \mathrm{C}$-trioctanoin absorption test is a breath test which measures the ${ }^{13} \mathrm{C}$ in expiratory carbon dioxide after a ${ }^{13} \mathrm{C}$-labeled compound is taken orally. 


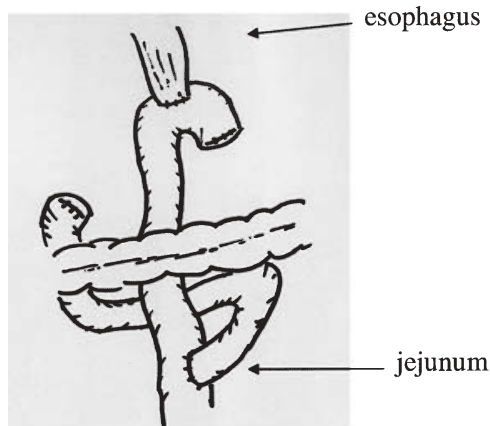

Roux-enY method

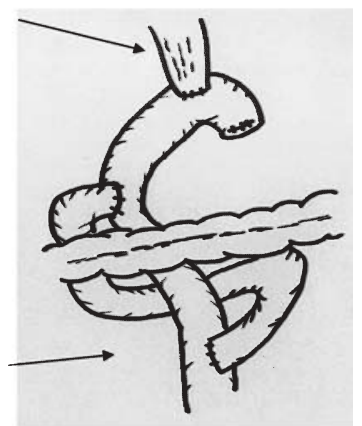

Double tract method
Fig. 1. Reconstruction methods after total gastrectomy

The double-tract (DT) reconstruction method and the Roux-en-Y (RY) method (Fig. 1) are performed as reconstruction methods after total gastrectomy. The DT reconstruction method is a reconstructive route in which food passes through the duodenum, so that bile acids and pancreatic juices are combined with the food, but the RY method has a different reconstructive route, in which all food passes through the jejunum, without passing through the duodenum. In the present study, the new examination was performed to identify a more physiological reconstructive method and thus obtain better absorption ability. The aim of this study was to evaluate the various reconstruction methods by comparing those with passage routes through the duodenum and those without, by a quantitative analysis using the ${ }^{13} \mathrm{C}$-trioctanoin absorption test. In other words, we evaluated the reconstructive methods based on fat absorption ability, using a new test; we also evaluated other digestive and absorptive abilities using a conventional test, in order to determine the usefulness of the ${ }^{13} \mathrm{C}$-trioctanoin absorption test.

\section{Patients and methods}

\section{Patients}

Among patients who had undergone gastrectomy for gastric cancer at Toho University Ohashi Hospital during the 7-year period from 1993 to 2000, 40 patients who had been operated on between 1 and 3 years previously and were under the age of 80 were evaluated. Regarding the reconstruction methods, 10 patients had received reconstruction by the DT method and 10 had received reconstruction by the RY method after a total gastrectomy (Fig. 1). Twenty patients who had had reconstruction by the Billroth I (BI) method after a distal gastrectomy formed the control group. The mean age was $60.6 \pm 13.1$ years in the DT group, $65.3 \pm 6.5$ years in the RY group, and $66.7 \pm 10.3$ years in the BI group; no significant differences in background factors were observed. Concerning the ${ }^{13} \mathrm{C}$-trioctanoin absorption test, we employed 10 volunteers as a healthy control group. Any patients with diabetes, hepatic dysfunction, respiratory diseases, and urinary diseases were excluded from the study. All patients had invasion that was no deeper than the muscularis propria, and patients with over $\mathrm{n} 2$ lymph node metastasis were also excluded. The degree of lymph node dissection was limited to D1 or D2 and only patients with curative operations (curability A) were selected. Furthermore, patients with pancreas and spleen resections, postoperative complications, or disease recurrence were also excluded.

\section{Methods}

\section{Weight change}

The patients' weights $(\mathrm{kg})$ were measured preoperatively, and postoperatively at 1, 3, 6, 9, and 12 months. The preoperative weight was regarded as $100 \%$ and the change of weight was calculated and evaluated.

\section{5-g Oral glucose tolerance test (75 g OGTT)/} immunoreactive insulin (IRI) test

Early in the morning after overnight fasting, the patients ingested $225 \mathrm{ml}$ of Trelan G75 (Shimizu Pharmaceutical, Tokyo, Japan). Blood tests were performed before, and at 30, 60, 90,120,150, and $180 \mathrm{~min}$ after the loading, and the blood sugar and blood insulin levels were measured.

\section{PFD test}

After overnight fasting, the patients ingested the PFD medicine. One hour after this, the patients drank more than $200 \mathrm{ml}$ of water. Thereafter, the excretion rate (\%) of paraaminobenzoic acid (PABA) in total urine was measured until $6 \mathrm{~h}$ after they had taken the PFD medicine.

\section{D-Xylose test}

Early in the morning after overnight fasting, the patients received $25 \mathrm{~g}(0.5 \mathrm{~g} / \mathrm{kg})$ D-xylose dissolved in $250 \mathrm{ml}$ water, orally administered. Two hours later, $250 \mathrm{ml}$ of water was additionally administered. At $5 \mathrm{~h}$ after the administration of D-xylose, a urine sample was taken and the amount of $\mathrm{D}$-xylose contained in the urine was measured.

\section{${ }^{13} \mathrm{C}$-Trioctanoin absorption test}

After 14-15h of fasting, since supper on the previous day, the patients received $1 \mathrm{mg} / \mathrm{kg} \cdot$ body weight (BW) of ${ }^{13} \mathrm{C}$-trioctanoin $\left(\left[\mathrm{CH}_{3}\left(\mathrm{CH}_{2}\right)_{6}{ }^{13} \mathrm{COOCH}_{2}\right]_{2} \mathrm{CHOO}^{13} \mathrm{C}\right.$ $\left.\left(\mathrm{CH}_{2}\right)_{6} \mathrm{CH}_{3}\right)$, orally administered with $10 \mathrm{~g}$ of butter as the loading food. The patients then breathed into a 250$\mathrm{ml}$ breath-test bag (Shiseido, Tokyo, Japan). This test 
was done seven times; before the administration and at $30,60,90,120,150$, and $180 \mathrm{~min}$ after the administration. After the patients had taken the ${ }^{13} \mathrm{C}$-trioctanoin, they were then asked to lie quietly in bed. The amount of ${ }^{13} \mathrm{CO}_{2}$ from the expired $\mathrm{CO}_{2}$ gas was analyzed with the Automated Breath ${ }^{13}$ Carbon Analyzer (ABCA)-G (Europe Scientific, Crewe, UK), using the gas chromatography-mass spectrometry (GC-MS) method. The measurement results were expressed as the value of $\Delta^{13} \mathrm{C}(\%)$ and the $\%{ }^{13} \mathrm{Cdose}(\%$ dose). The calculation methods were as follows:

$$
\begin{aligned}
& \delta^{13} \mathrm{C}(\% \mathrm{o})=\frac{\mathrm{R}_{\text {sample }}-\mathrm{R}_{\text {standard }}}{\mathrm{R}_{\text {standard }}} \times 1000 \\
& \mathrm{R}_{\text {sample }}=\left({ }^{13} \mathrm{CO}_{2} /{ }^{12} \mathrm{CO}_{2}\right)_{\text {sample }} \\
& \mathrm{R}_{\text {standard }}=\left({ }^{13} \mathrm{CO}_{2} /{ }^{12} \mathrm{CO}_{2}\right)_{\text {standard }}
\end{aligned}
$$

For the standard, Pee Dee Bermnite (National Institute of Standard and Technology [NIST]; Gaithersburg, MD, USA) was used.

$$
\Delta^{13} \mathrm{C}(\% \mathrm{o})=\left(\delta^{13} \mathrm{C}\right)_{t}-\left(\delta^{13} \mathrm{C}\right)_{0}=\frac{(\mathrm{R})_{t}-(\mathrm{R})_{0}}{\mathrm{R}_{\text {standard }}} \times 1000
$$

After Eq. A was calculated, according to the $\delta^{13} \mathrm{C}$ levels before and after the administration of ${ }^{13} \mathrm{C}$-trioctanoin (at $t$ min) in the $\mathrm{CO}_{2}$ breath test, the $\Delta^{13} \mathrm{C}$ level was calculated.

$$
\begin{aligned}
\%\left({ }^{13} \mathrm{C}\right) \text { dose } \\
=\sum_{\mathrm{t}=\mathrm{t}_{0}}^{\mathrm{t}=\mathrm{t}_{1}} \frac{\Delta^{13} \mathrm{C} \times\left({ }^{13} \mathrm{CO}_{2} /{ }^{12} \mathrm{CO}_{2}\right) \text { standard } \times \mathrm{MW} \times 10}{\text { Dose } \times \# \times \mathrm{APE}} \\
\quad \times \mathrm{CO} 2 \text { production }
\end{aligned}
$$

where $\mathrm{MW}$ is the molecular weight of ${ }^{13} \mathrm{C}$-trioctanoin; \# is the number of ${ }^{13} \mathrm{C}$ within a molecule; APE is the concentration of ${ }^{13} \mathrm{C}$ (atomic \% excess); and $\mathrm{CO} 2$ production $=$ body surface area $($ BSA $) \times 300$

Statistical evaluation was done with Student's $t$-test or the Mann-Whitney $U$-test, and probabilities of less than 0.05 were accepted as significant.

\section{Results}

Weight change between the preoperative and postoperative measurements

The weight in the BI group at 1 month after surgery had significantly decreased, to $90.1 \pm 6.0 \%$ of the preoperative weight $(P<0.001 ;$ Fig. 2$)$. After the third month, no remarkable change was observed. In the DT group, the weight decreased to $87.0 \pm 10.4 \%$ at 1 month postoperatively, but the weight then increased from month 6 to month 12. The weight was $90.5 \pm 9.1 \%$ of the preoperative weight at month 12 . In the RY group, the weight demonstrated a gradual decrease from $86.9 \pm$ $7.4 \%$ at the first month postoperatively to $82.9 \pm 3.7 \%$ (preoperative value vs the value at postoperatively month $12 ; P<0.001)$ at 12 months postoperatively. At month 12 , postoperatively, significant differences were observed between the BI group and the RY group $(P<$ $0.01)$, and between the DT group and the RY group $(P<0.05)$.

\section{5-g OGTT and IRI test}

In the 75-g OGTT, for the levels prior to the test, there were no significant differences among the three groups, and each level was normal at fasting (Table 1). In the RY group, the peak level was more than $250 \mathrm{mg} / \mathrm{dl}$ at $30 \mathrm{~min}$. The RY group showed more marked changes

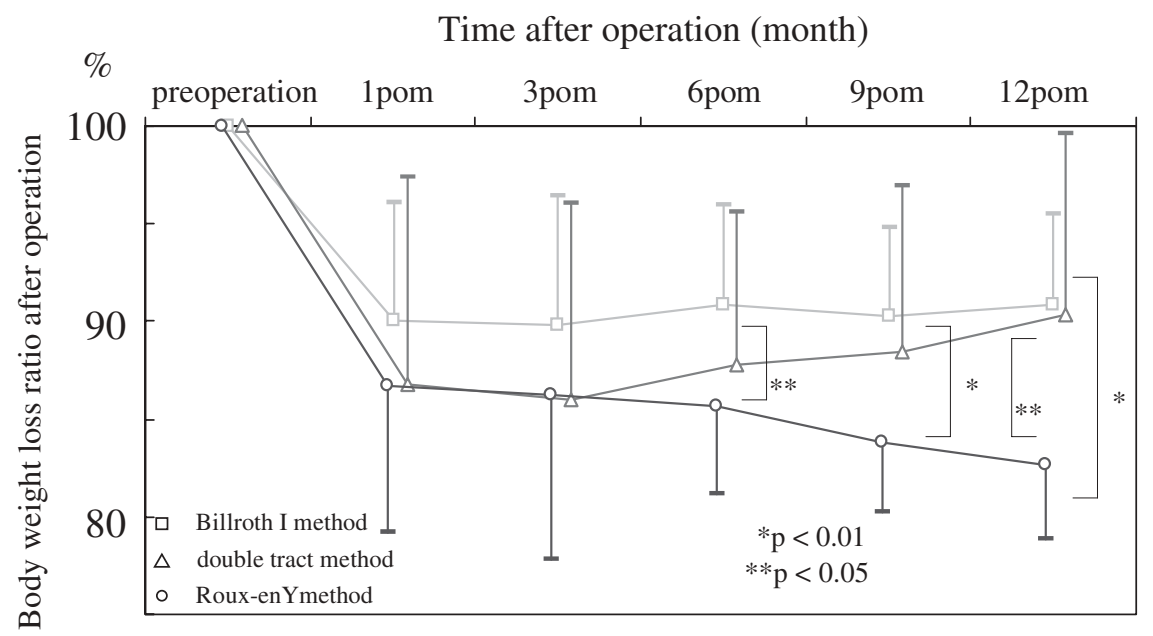

Fig. 2. Weight change after gastrectomy. Postoperative weight showed an increase in the double-tract group from 3 months after the operation, and a decrease in the Roux-en-Y group. pom, postoperative month 
Table 1. Comparison of 75-g oral glucose tolerance test (OGTT) and immunoreactive insulin (IRI) test results according to each of the reconstructions

\begin{tabular}{|c|c|c|c|c|c|}
\hline & Minutes & Billroth I (BI) & Double-tract (DT) & Roux-en-Y (RY) & $P$ value \\
\hline \multirow[t]{7}{*}{ 75-g OGTT (mg/dl) } & 0 & $86.3 \pm 11.1$ & $89.1 \pm 6.2$ & $88.4 \pm 8.8$ & NS \\
\hline & 30 & $176.0 \pm 38.8(1 * ; 2 *)$ & $214.2 \pm 28.6(1 *)$ & $253.8 \pm 47.7(2 *)$ & $1 * P=0.017 ;{ }^{2 *} P<0.001$ \\
\hline & 60 & $167 \pm 61.0\left(3^{*} ; 4^{*}\right)$ & $229.1 \pm 46.1\left(3^{*}\right)$ & $239.8 \pm 41.3(4 *)$ & $3 * P=0.015 ;{ }^{4 *} P=0.002$ \\
\hline & 90 & $124.4 \pm 58.9\left(5^{*}\right)$ & $182.0 \pm 46.8\left(5^{*}\right)$ & $159.4 \pm 72.3$ & $*^{5} P=0.02$ \\
\hline & 120 & $92.3 \pm 51.3$ & $121.1 \pm 44.8$ & $103.3 \pm 63.8$ & NS \\
\hline & 150 & $81.2 \pm 41.2$ & $84.3 \pm 33.4$ & $76.9 \pm 44.3$ & NS \\
\hline & 180 & $82.5 \pm 42.0$ & $67.8 \pm 23.7$ & $71.9 \pm 23.0$ & NS \\
\hline \multirow[t]{7}{*}{$\operatorname{IRI}(\mu \mathrm{U} / \mathrm{ml})$} & 0 & $5.4 \pm 2.1$ & $4.9 \pm 2.5$ & $4.2 \pm 1.3$ & NS \\
\hline & 30 & $61.3 \pm 42.4$ & $51.1 \pm 20.6$ & $53.5 \pm 22.3$ & NS \\
\hline & 60 & $68.8 \pm 48.5$ & $78.8 \pm 30.0$ & $96.0 \pm 43.3$ & NS \\
\hline & 90 & $42.8 \pm 32.2$ & $65.0 \pm 45.8$ & $68.4 \pm 53.8$ & NS \\
\hline & 120 & $24.9 \pm 16.5$ & $26.9 \pm 20.1$ & $30.2 \pm 28.2$ & NS \\
\hline & 150 & $14.6 \pm 11.5$ & $12.8 \pm 7.8$ & $19.2 \pm 16.2$ & NS \\
\hline & 180 & $9.1 \pm 5.2$ & $7.2 \pm 2.8$ & $7.4 \pm 5.3$ & NS \\
\hline
\end{tabular}

In the 75-g OGTT, the Roux-en-Y group, in which food is allowed to enter directly into the jejunum, showed higher values than the other two groups. In the IRI test, there was no significant difference between the groups, but the Roux-en-Y group had a higher value. NS, not significant

Table 2. Comparison of pancreatic function diagnostic (PFD) and D-xylose test results for each of the reconstructions

\begin{tabular}{lccc}
\hline & PFD (\%) & D-xylose (g/l) & $P$ value \\
\hline Billroth I method & $92.7 \pm 16.4$ & $6.4 \pm 1.7$ & NS \\
Double-tract method & $93.4 \pm 25.4$ & $6.9 \pm 2.2$ & NS \\
Roux-en-Y method & $91.3 \pm 18.5$ & $5.9 \pm 1.7$ & NS \\
\hline
\end{tabular}

The findings were all in the normal range and no significant differences were observed

than the other groups. At $30 \mathrm{~min}$, there were significant differences between the BI group and both the DT group and the RY group $(P=0.017 ; P<0.001)$. In addition, at $60 \mathrm{~min}$, significant differences were observed between the BI group and both the DT group and the RY group $(P=0.015 ; P<0.01)$. Furthermore, at $90 \mathrm{~min}$, there was a significant difference between the BI group and the DT group $(P=0.02)$.

In the IRI test, for the levels prior to the test, there was no significant difference between the groups at fasting, and each level was normal. For all reconstructive procedures, the highest level was observed at $60 \mathrm{~min}$. Similar to the result of the 75-g OGTT in the RY group, the highest levels tended to be observed when food passed through the small intestine. However, there was no significant difference after the highest levels were observed.

\section{PFD test and D-xylose test}

In the PFD test and D-xylose test, all levels were in the normal range (PFD, $>70 \%$; D-xylose, 4.1-8.2 g/l), and there were no significant differences between the groups (Table 2). The results of the PFD test according to the reconstruction procedure were $92.7 \pm 16.4 \%$ in the BI group, $93.4 \pm 25.4 \%$ in the DT group, and $91.3 \pm$ $18.5 \%$ in the RY group; the level of the RY group tended to be low. In the D-xylose test, even though the level tended to be higher in the DT group $(6.9 \pm 2.2 \mathrm{~g} / \mathrm{l})$ the values for the the BI group and the RY group were $6.4 \pm 1.7 \mathrm{~g} / \mathrm{l}$ and $5.9 \pm 1.7 \mathrm{~g} / \mathrm{l}$, respectively.

\section{${ }^{13} \mathrm{C}$-Trioctanoin absorption test}

$\Delta^{13} \mathrm{C}(\%)$ level

The $\Delta^{13} \mathrm{C}(\%$ ) level prior to the test was regarded as $0 \%$, and the change in the degree of absorption after ingestion was observed every $30 \mathrm{~min}$. In healthy controls, the level showed a higher increase than in patients after gastrectomy (Fig. 3). The level peaked at $7.7 \pm 2.1 \%$ at $90 \mathrm{~min}$ and at $7.7 \pm 2.3 \%$ at $120 \mathrm{~min}$. Thereafter, the level declined until $180 \mathrm{~min}$. In the BI group, the level increased and reached the highest point of $7.6 \pm 2.8 \%$ o at $90 \mathrm{~min}$, and thereafter the level gradually decreased. In the DT group, the increase was slower; the level peaked at $7.1 \pm 3.0 \%$ at $120 \mathrm{~min}$. Afterward, the course of change was the same as that in the BI group. In the RY group, the level recorded a peak of $5.5 \pm 3.3 \%$. The level increased more slowly than the levels in the healthy controls, BI group, and DT group. There were significant differences from these groups at $60 \mathrm{~min}$ and $90 \mathrm{~min}$. In the RY group, the level at $60 \mathrm{~min}$ was $2.0 \pm$ $2.1 \%$, which was significantly lower than the level for the healthy controls $(P=0.005)$, the BI group $(P=$ $0.026)$, and the DT group $(P=0.045)$. Furthermore, at $90 \mathrm{~min}$, the level in the RY group was lower, at $3.5 \pm$ $2.5 \%$, with a significant difference from the healthy controls $(P<0.001)$, the BI group $(P<0.001)$, and the DT group $(P=0.0098)$. At $120 \mathrm{~min}$, there was a significant difference between the DT group $(5.4 \pm 3.1 \%$ o $)$ and the 


\section{$\%$}

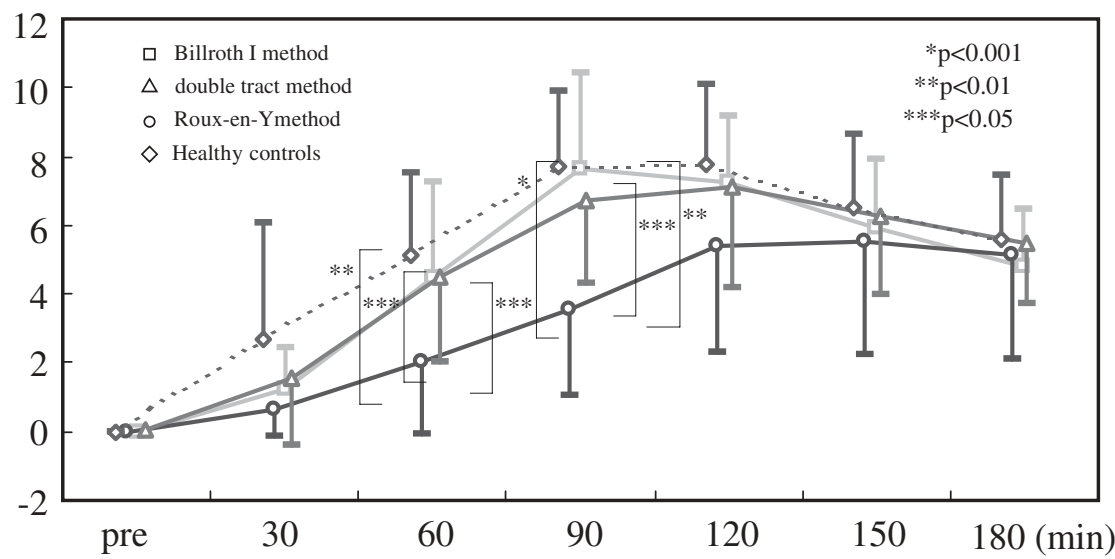

Fig. 3. Time course of ${ }^{13} \mathrm{C}$-trioctanoin breath-test findings after gastrectomy. In the ${ }^{13} \mathrm{C}$-trioctanoin absorption test, the Billroth I group and the double-tract group showed a similar course to that of the healthy controls. The Roux-en-Y group showed lower values

Table 3. Comparison of the cumulative \%dose of ${ }^{13} \mathrm{C}$ trioctanoin for each of the reconstructions

\begin{tabular}{lllcccc}
\hline & 0-30 Min & 0-60 Min & 0-90 Min & $0-120$ Min & $0-150$ Min & 0-180 Min \\
\hline Billroth I method (\%) & $1.0 \pm 0.8^{* * 1}$ & $3.1 \pm 1.9^{* * 3}$ & $7.7 \pm 3.2^{* 2}$ & $13.5 \pm 4.1^{* 4}$ & $18.6 \pm 4.9^{* 5}$ & $22.8 \pm 5.7^{* * 8}$ \\
Double-tract method (\%) & $1.1 \pm 1.3$ & $3.3 \pm 2.7$ & $7.5 \pm 3.8^{* * 6}$ & $12.8 \pm 5.4^{* * 7}$ & $17.8 \pm 6.8$ & $22.2 \pm 7.8$ \\
Roux-en-Y method (\%) & $0.6 \pm 1.0^{* * 2}$ & $1.7 \pm 1.8^{* 1}$ & $4.1 \pm 3.4^{* 3}$ & $7.9 \pm 5.2^{* * * 1}$ & $12.3 \pm 7.2^{* 6}$ & $16.5 \pm 9.3 * 7$ \\
Healthy control (\%) & $2.8 \pm 2.9$ & $5.8 \pm 4.7$ & $10.6 \pm 5.4$ & $16.2 \pm 5.3$ & $21.5 \pm 5.5$ & $26.0 \pm 5.9$
\end{tabular}

* $P<0.01 ; * * P<0.05 ; * * * P<0.001$

*1: RY VS HC, *2: BI VS RY, *3: RY VS HC, *4: BI VS RY, *5: BI VS RY, *6: RY VS HC, *7: RY VS HC, **1: BI VS HC, **2: RY VS HC, $* * 3$ : BI VS HC, **4: BI VS RY, **5: BI VS HC, **6: DT VS RY, **7: DT VS RY, **8: BI VS RY, ***1: RY VS HC

Regarding the cumulative \%dose, there was a significant difference between the Roux-en-Y group and the other groups

HC, healthy control

healthy control group $(P=0.043)$. When comparing the averages of the peak levels, that for the healthy controls was $8.9 \pm 1.9 \%$, for the BI group it was $8.5 \pm 2.1 \%$, for the DT group it was $7.6 \pm 2.6 \%$, and that for the RY group was $5.9 \pm 3.4 \%$; the values for the healthy controls $(P=0.006)$ and the BI group $(P=0.007)$ were higher than that for the RY group, and the differences were significant. The value for the DT group tended to be higher than that for the RY group, but there were no significant differences $(P=0.086)$. In terms of the average time at peak level, that for the healthy controls was $90.0 \pm 32.9 \mathrm{~min}$, for the BI group, it was $93.5 \pm 20.9 \mathrm{~min}$, for the DT group it was $108.0 \pm 29.0 \mathrm{~min}$, and for the RY group it was $139.1 \pm 30.8 \mathrm{~min}$; trioctanoin absorption was slow for the reconstructive procedures that allowed food to directly enter the jejunum. The RY group was slower than the healthy controls $(P=0.002)$, the BI group $(P<0.001)$, and the DT group $(P=$ 0.028 ), and the differences were significant.

\section{$\%$ dose}

The \%dose is the absorption ratio against the ingested dose of ${ }^{13} \mathrm{C}$-trioctanoin. In the cumulative \%dose each $30 \mathrm{~min}$, a significant difference was seen between the RY group and the healthy control group for all periods
(Table 3). In the BI group, there was a significantly higher level compared with the RY group after $90 \mathrm{~min}$. In addition, a significant difference was also seen between the DT group and the RY group at $90 \mathrm{~min}$ and $120 \mathrm{~min}$. At $3 \mathrm{~h}$, the levels were $22.8 \pm 5.7 \%$ in the $\mathrm{BI}$ group, $22.2 \pm 7.8 \%$, in the DT group, and $16.5 \pm 9.3 \%$ in the RY group; with a value of $26.0 \pm 5.9 \%$ in the healthy control group. The \%dose was less in the RY group (in which food is allowed to directly pass into the jejunum) than in the BI group, and a significant difference was seen at $3 \mathrm{~h}$. But no significant difference was observed between the RY group and the DT group. There was a significant difference between the RY group and the healthy control group $(P=0.007)$. In comparisons of the cumulative \%dose, in the RY group fat absorption was clearly decreased in comparison to that in the healthy control group, the BI group, and the DT group.

\section{Discussion}

Conventionally, various examinations have been performed including fat digestion and absorption test [1-4]. In 1962, a digestion and absorption study of fat in which 
${ }^{14} \mathrm{C}$-labeled lipid was administered and ${ }^{14} \mathrm{CO}_{2}$ from expired gas was measured was first reported by Schwabe et al. [5]. Subsequently, many authors [6-8] have described the digestion and absorption of fat, using ${ }^{14} \mathrm{C}$. However, because ${ }^{131}$ I-labeled compounds and ${ }^{14} \mathrm{C}$ labeled lipids are radioactive isotopes and may harm patients, their usage has decreased. Since the 1970s, the ${ }^{13} \mathrm{CO}_{2}$ test of expired gas by ${ }^{13} \mathrm{C}$-labeled compounds has been adopted [9-11]. Someya et al. [12] and Sasaki [13] reported ${ }^{13} \mathrm{C}$ and ${ }^{14} \mathrm{C}$ breath tests. ${ }^{13} \mathrm{C}$ is described as a stable isotope, and it is reported to share $1.1 \%$ of existing carbon atoms in nature. As it does not contain radioactivity and it does not have any possibility of explosion or contamination, it can be safely used for children and pregnant women. However, up to now, breath tests utilizing ${ }^{13} \mathrm{C}$-labeled compounds have not been widely used, because ${ }^{13} \mathrm{C}$-labeled compounds are expensive and require a specific analyzer for measurement. We found new quantitative results after gastrectomy using the ${ }^{13} \mathrm{C}$ trioctanoin breath test.

To evaluate digestion and absorption ability after gastrectomy, conventional examinations, such as the PFD test, D-xylose test, and ${ }^{131}$ I compound test have also been employed. However, the PFD test and D-xylose test are not direct examinations. Moreover, as the measurements from these tests are based on the amount of evacuated urine, it could make the diagnosis difficult in patients with urinary tract diseases, such as renal insufficiency. Especially for aged persons, urinary tract abnormalities are observed in many people, making the diagnosis difficult. In terms of examinations utilizing ${ }^{131}$ I compounds, as evacuated stool is used for the test, it is difficult to gain constant and reliable results because of the unequal condition of stools and the complexity of the examination itself. Therefore, this test cannot be a generally accepted one. There have, so far, been no reports which evaluated reconstructive operations after gastrectomy by direct examinations utilizing ${ }^{14} \mathrm{C}$ or ${ }^{13} \mathrm{C}$ compounds.

The present study was a comparative study utilizing direct digestion and absorption tests. In this study, we used ${ }^{13} \mathrm{C}$-trioctanoin, a medium-chain triglyceride, trioctanoin, that is a ${ }^{13} \mathrm{C}$-labeled lipid. Medium-chain triglycerides are triglycerides that consist of fatty acids that have 6-12 carbon chains, and glycerin. Lipids most likely cause digestion and absorption disturbances. In particular, medium-chain triglycerides are different from long-chain fats, because they show a fast absorption peak and large mass $[10,14]$. In other words, a test with medium-chain triglycerides takes a shorter time and gives more accurate results. Medium-chain triglycerides are mostly absorbed in the small intestine as medium-chain triglyceride acid and then through the portal vein are carried into the liver. Absorption has been reported to be closely related to bile acids [15] and to pancreatic exocrine function [16]. The DT and BI groups have reconstruction routes in which food passes through the duodenum, which thus allows bile acids and pancreatic juice to combine with food. The RY group has a different reconstructive route, in which food does not pass through duodenum. Comparing these reconstructive routes is considered to be a feasible way to evaluate the nutrition in patients with each gastric reconstructive procedure after gastrectomy.

In our comparisons of weight (body weight) loss after these reconstructive procedures, the BI group had the lowest weight change, and these patients maintained about $90 \%$ of their previous weight. The DT group had weight loss until 3 months after the operation, but the weight had recovered to about $90 \%$ of the previous weight at 12 months after the surgery, and thus was as high as the level in BI group. For this reason, the patients who underwent the BI and DT methods recovered better after gastrectomy than the patients who underwent the RY method. After all, the DT method is a more physiologic reconstruction method than the RY method after a total gastrectomy. The results were based not only on the range of resection but also on the fact that a difference in the reconstructive procedures could influence the patients' postoperative nutrition. We assumed that the reconstructions would demonstrate different digestive and absorptive function measured by the various tests performed. In terms of the 75-g OGTT, although patients with RY have been reported to have a glucose tolerance disturbance, the peak level at $30 \mathrm{~min}$ was significantly higher in the RY group. Results for the IRI test showed the same tendency, and the level was higher in the groups without food passage through the duodenum. This may be caused by the direct influx of carbohydrates into the small intestine. In the RY group, a more rapid absorption of glucose occurs in comparison with other reconstructions. The PFD test is affected by pancreatic exocrine function, and the D-xylose test is affected by the area for absorption in the small intestine. There was no significant difference in the PFD and D-xylose test results between the reconstructive procedures. This is because pancreatectomy with expanded lymph node dissection was excluded. Also, these results indicated that the pulled-up small intestine for the esophagojejunostomy had no influence on the absorptive area in the jejunum.

In previous reports, conventional tests showed no significant difference between reconstructions with and without food passage through duodenum. Therefore, we investigated reconstructions after gastrectomy using the ${ }^{13} \mathrm{C}$-trioctanoin breath test. The ${ }^{13} \mathrm{C}$-trioctanoin breath test is a new test which has not been previously performed after gastrectomy. The ${ }^{13} \mathrm{C}$-trioctanoin test showed, according to the reconstruction, that the aver- 
age of the peak of $\Delta^{13} \mathrm{C}$ was highest in the $\mathrm{BI}$ group, followed by the DT group; the procedures in which food passes through the duodenum tended to show a high level. The average time of peaking in the BI and DT groups and the healthy controls was significantly shorter than that in the RY group. Regarding the $\Delta^{13} \mathrm{C}$ duration, at an early stage after administration, the BI and DT groups and the healthy controls showed a significantly higher level than the RY group contrary to the results of the 75-g OGTT test. Both the healthy controls and reconstructions besides RY showed steep rises in $\Delta^{13} \mathrm{C}$ duration. In other words, procedures which allowed food to pass through the duodenum resulted in a considerably more rapid absorption of medium-chain triglyceride and showed a high peak $\Delta^{13} \mathrm{C}$ level, at almost the same level as the healthy controls. In the group without food passage though the duodenum, the $\Delta^{13} \mathrm{C}$ level increased at a late stage, because in the jejunum bile acid and pancreatic juice combine with the food after the food passes through the duodenal anastomosis.

Regarding the \%dose, which indicates the absorption amount compared with the ingestion amount, it was higher in the BI and DT groups and healthy controls than in the RY group. As the BI and DT groups showed no significant difference in comparison to the healthy control group in this study, they are thus considered to be more similar to normal and more physiological reconstructive methods. In the weight recovery of patients after gastrectomy, we found that fat absorption influenced nutrition after operation. In other words, reconstruction without food passage through the duodenum after gastrectomy should be avoided, and a physiological reconstruction should be selected, with food passage through the duodenum, whenever possible.

In the choice of reconstructions, digestion and absorption disturbances, resulting in weight loss after gastrectomy, remain a problem. Concerning the function of digestion and the absorption of sugars after gastrectomy, all of the reconstructive procedures we examined here demonstrated little disturbance. With regard to the function of digestion and the absorption of protein, many aspects remain controversial, and no definitive conclusions have yet been made. In contrast, with regard to digestion and the absorption of lipids, many reports have concluded that physiological procedures which allow the passage of food through the duodenum tend to be more favorable, but without any significant difference between procedures [17-19]. Up to now, medium-chain triglycerides have been considered to be absorbed in the duodenum according to the action of bile acids and pancreatic lipase. In this study, the absorption of medium-chain triglycerides was affected by the passage of food through the duodenum. In addition, medium-chain triglycerides were absorbed to a greater extent and more quickly after being combined with bile acids and pancreatic lipase. As an evaluation of the function of digestion and absorption based on gastric reconstructive procedures, this new method, utilizing ${ }^{13} \mathrm{C}$-trioctanoin, was employed to evaluate the function of digestion and the absorption of fat directly. There have, so far, been no reports which described the absorption process over the course of time. In the light of our findings, our way of testing is considered to be extremely feasible. In reconstructions without the passage of food through the duodenum after gastrectomy, the recovery of weight was worse, and the absorption of medium-chain triglycerides was lower than that in healthy controls. A reconstruction method which allows the passage of food through the duodenum should thus be selected whenever possible.

Acknowledgments We would like to especially thank Professor Sadahito Usui, Professor, Third Department of Surgery, Toho University, who gave us tremendous advice and encouragement, and Dr. Yoshihisa Saida, Lecturer, Third Department of Surgery, Toho University, who helped us to make this study a reality. In addition, we would also like to extend our deepest appreciation to all the doctors from the digestive surgery group of this department for their great help and cooperation.

\section{References}

1. Rutenberg AM, Seligman AM, Fine J. Studies with radioactive iodized fat. I. Preparation of radioactive fat with observations on the absorption of fat following subcutaneous and intraperitoneal injection in dogs. J Clin Invest 1949;28:1105-9.

2. Stanley MM, Thannhauser SJ. The absorption and disposition of orally administered ${ }^{131}$-labeled neutral fat in man. J Lab Clin Med 1949;34:1634-9.

3. Thannhauser SJ, Stanley MM. Serum fat curves following oral administration of $\mathrm{I}^{131}$-labeled neutral fat to normal subjects and those with idiopathic hyperlipemia. Tr A Am Physician 1949; 62:245-52.

4. Nakamoto T. Standard methods of ${ }^{131} \mathrm{I}$-triolein test and ${ }^{131} \mathrm{I}$-oleic acid test for fat absorption study, and their clinical investigation (in Japanese with English abstract). Jpn J Gastroenterol 1968; 65:363-82.

5. Schwabe AD, Cozzetto FJ, Bennett LR, Mellinkoff SM. Estimation of fat absorption by monitoring of expired radioactive carbon dioxide after feeding a radioactive fat. Gastroenterology 1962;42: 285-91.

6. Abt AF, Von Schuching SL. Fat utilization test in disorders of fat metabolism. A new diagnostic method applied to patients suffering with malabsorption syndrome, chronic pancreatitis, and arteriosclerotic cardiovascular disease. Bull Johns Hopkins 1966;119: 316-30.

7. Kaihara S, Wagner HN. Measurement of intestinal fat absorption with carbon-14 labeled tracers. J Lab Clin Med 1968;71:400-11.

8. Burrows PJ, Fleming JS, Garnett ES, Ackery DM, Colin-Jones DG, Bamforth J. Clinical evaluation of the ${ }^{14} \mathrm{C}$ fat absorption test. Gut 1974;15:147-50.

9. Schoeller DA, Schnider JF, Solomons NW, Watkins JB, Klein $\mathrm{PD}$. Clinical diagnosis with the stable isotope ${ }^{13} \mathrm{C}$ in $\mathrm{CO}_{2}$ breath 
tests: methodology and fundamental considerations. J Lab Clin Med 1977;90:412-21.

10. Watkins JB, Schoeller DA, Klein PD, Ott DG, Newcomer AD, Hofmann AF. ${ }^{13} \mathrm{C}$-Trioctanoin, a nonradioactive breath test to detect fat malabsorption. J Lab Clin Med 1977;90:422-30.

11. Watkins JB, Klein PD, Schoeller DA, Kirschner BS, Park R, Perman JA. Diagnosis and differentiation of fat malabsorption in children using ${ }^{13} \mathrm{C}$-labeled lipids: trioctanoin, triolein, and palmitic acid breath tests. Gastroenterology 1982;82:911-7.

12. Someya K, Sasaki Y, Oh-hara H, Maeda T. Animal model for the comparative study of ${ }^{14} \mathrm{C}$ - and ${ }^{13} \mathrm{C}$-breath tests (in Japanese with English abstract). St. Marianna Med J 1976;4:530-5.

13. Sasaki Y. Clinical application of breath test with isotopes of carbon (in Japanese with English abstract). J Med Soc Toho 1983;29:559-67.

14. Lossow WJ, Chaikoff IL. Carbohydrate sparing of fatty acid oxidation. I. The relation of fatty acid chain length to the degree of sparing. II. The mechanism by which carbohydrate spares the oxidation of palmitic acid. Arch Biochem 1955;57:23-40.

15. Kashima K. Digestion and absorption of medium chain triglyceride (MCT), in vivo and in vitro, in comparison with long chain triglyceride (LCT) (in Japanese with English abstract). Jpn J Gastroenterol 1970;67:1082-100.

16. Sakamoto A, Usui S, Toyoizumi S, Takahashi S, Nabeya Y, Okuyama K, et al. Clinical evaluation of fat digestion and absorption by means of stable isotopes after pancreatoduodenectomy (in Japanese with English abstract). Jpn J Gastroenterol Surg 1990;23:47-51.

17. Misumi A, Akagi M, Baba K, Misumi K, Shojima T, Ohtsu S, et al. Problems in surgical treatments of carcinoma of the gastric cardia; comparison between proximal gastrectomy and total gastrectomy (in Japanese with English title). Jpn J Gastroenterol Surg 1984; 17:6-14.

18. Hamanaka Y, Ueno T, Yamamoto T, Suzuki T. A new diagnostic method for fat indigestion and malabsorption and clinical usefullness (in Japanese with English title). JJPEN (Jpn J Parenter Enter Nutr) 1992;14:3-8.

19. Momose R. Study of bone metabolism, intestinal absorption and anemia in long courses after total gastrectomy (in Japanese with English abstract). Jpn J Gastroenterol Surg 1991;24:779-87. 